\title{
Cycling Through Migraine Preventive Treatments: Implications for All-Cause Total Direct Costs and Disease-Specific Costs
}

\author{
Janet H. Ford, MPH, PhD; Krista Schroeder, PhD; Allen W. Nyhuis, MS; \\ Shonda A. Foster, PharmD; and Sheena K. Aurora, MD
}

\begin{abstract}
BACKGROUND: Migraine is a common and disabling neurological disease associated with substantial economic burden. Among patients with migraine, it is unknown if cost differences exist when preventive migraine medication (PMM) switches occur.
\end{abstract}

OBJECTIVE: To understand the cost burden and health care resource utilization of patients who discontinue or cycle through 1 (PMM1), 2 (PMM2), or $\geq 3$ (PMM3) unique PMM drug classes over a 12-month period versus patients who adhere persistently to their initial PMM class.

METHODS: This retrospective observational study used the Truven Health Analytics MarketScan databases to identify adult patients with migraine initiating their first PMM class (antidepressants, antiepileptics, beta blockers, or neurotoxins) from 2011-2013 (index date $=$ first PMM claim). Patients were required to have $\geq 2$ outpatient ( 1 if inpatient) migraine diagnosis codes (ICD-9-CM 346.xx) from 1 year pre-index to 1 year post-index with $\geq 1$ code occurring pre-index. Inclusion criteria also required 12 months of pre- and post-index continuous medical and prescription enrollment. All-cause and migraine-specific total direct costs (outpatient, inpatient, emergency department, and prescriptions), based on the 2014 Consumer Price Index, were estimated for each PMM versus a persistent subgroup during the 12-month post-index period. Propensity score bin bootstrapping, controlling for patient baseline characteristics, was used to adjust separate cost comparisons between each PMM subgroup and the persistent subgroup; bootstrap simulations yielded propensity score-adjusted $P$ values.

RESULTS: The study population included 55,402 patients who received a PMM. The study population was mainly female $(85 \%)$ with a mean age of 39.2 years and mean Charlson Comorbidity Index of 0.31 . Antiepileptics were the most common drug class chosen at index across all subgroups; however, lower use of antiepileptics was observed in PMM2 and PMM3 subgroups, which were more likely to be prescribed either antidepressants or beta blockers at index. Mean all-cause total direct costs, including prescription costs, were significantly higher in PMM2 $(\$ 13,429)$ and PMM3 $(\$ 18,394)$ subgroups versus the persistent subgroup $(\$ 11,941$; each adjusted pairwise comparison, $P<0.001)$. Mean migraine-specific total direct costs were significantly lower for the persistent subgroup $(\$ 2,420)$ versus PMM2 and PMM3 subgroups and escalated with increasing numbers of drug class discontinuations or switches, from a mean of $\$ 2,997$ to $\$ 5,004$ (both adjusted pairwise comparisons, $P<0.001$ ). Subgroup differences in all-cause and migraine-specific direct costs were primarily due to variations in outpatient and emergency department services.

CONCLUSIONS: All-cause total direct costs rose with increasing number of PMM switches over the 12-month post-index period, and were significantly higher than in the persistent subgroup, with the exception of PMM1. Additional analyses indicated that the lack of increase between PMM-persistent and PMM1 costs was due to higher pharmacy costs that were likely related to continuous use of medication in the PMM-persistent subgroup. These data suggest an increased cost burden among patients with migraine who cycle through $\geq 2$ PMMs versus those who continue to receive their initial medication class.

J Manag Care Spec Pharm. 2019;25(1):46-55

Copyright $\odot 2019$, Academy of Managed Care Pharmacy. All rights reserved.

\section{What is already known about this subject}

Migraine is known to be a disabling, common neurological disease; despite its widespread nature, preventive medications are underused.

The currently available preventive medications for migraine are associated with intolerance, which frequently leads to switching and discontinuation.

Total direct annual health care costs are higher among patients with migraine compared with those without migraine, particularly as related to outpatient and emergency department services.

\section{What this study adds}

Higher health care resource utilization was observed among the subgroups with more preventive migraine medication switches or discontinuations.

Regardless of the first migraine preventive treatment choice at index, most patients made at least 1 switch or discontinued treatment within a year $(77.1 \%)$, with only $22.9 \%$ remaining persistent in their treatment.

Acute medication use, especially opioids, was higher among the subgroups with more preventive migraine medication switches or discontinuations during the post-index period.

T $\mathrm{n}$ the United States, estimated unadjusted total health care expenditures among people with migraine is $\$ 56.31$ billion per year; adjusted incremental total direct health care expenditure for those with migraine versus those without migraine is $\$ 9.21$ billion per year. ${ }^{1}$ Migraine is a common and disabling neurological disease, affecting nearly $12 \%$ of the U.S. population. ${ }^{2}$ Despite the availability of new U.S. treatment guidelines, ${ }^{3,4}$ only an estimated $13 \%$ of all patients with migraine receive any preventive therapy. ${ }^{5}$ The pathophysiology of migraine is complex and not well understood; however, recent advances show promise for new pharmacologic and 
behavioral interventions based on a better understanding of the mechanisms of migraine. ${ }^{6}$

Migraine is associated with substantial public health burden due to its high prevalence and prominent disability. ${ }^{7,8}$ The vast majority of direct costs are attributed to outpatient services (medications, office or clinic visits, emergency department [ED] visits, laboratory and diagnostic services, and management of treatment side effects). ${ }^{7}$ Although research has indicated that the use of preventive migraine medications (PMMs) decreases patient burden and reduces health care resource utilization (HCRU), ${ }^{9}$ currently available PMMs have been shown to induce side effects that can lead to multiple PMM switches, poor adherence, or discontinuation, resulting in many patients relying solely on acute medications for migraine relief. ${ }^{10-13}$

Among patients with migraine, it is unknown if cost and HCRU differences exist when PMM switches occur. Accordingly, the primary aim of this study was to understand (a) all-cause and migraine-specific direct costs and the HCRU burden of patients who are exposed to or discontinue 1, 2, or 3 or more PMM classes within a 12-month time period and (b) the relevant treatment patterns associated with these 3 subgroups compared with patients who are persistent on their initial PMM class. A secondary aim was to describe acute pain medication use (opioids, barbiturates, triptans, nonsteroidal anti-inflammatory drugs [NSAIDs], ergot alkaloids, and isometheptenes) across the PMM subgroups during the post-index period.

\section{Methods}

\section{Study Design and Data Sources}

This retrospective observational database study extracted and analyzed claims data from the Truven Health Analytics MarketScan databases. The Truven patient population consists of more than 240 million unique commercially insured patients, covered dependents, and retirees with employersponsored supplemental Medicare coverage. These data contain de-identified administrative claims capturing patient-level data on age, gender, geographic region, and HCRU; expenditures; and enrollment across inpatient, outpatient, prescription drug, and carve-out services. These databases link paid claims and encounter data, capturing when services occurred and diagnosis codes via the International Classification of Diseases, Ninth Revision, Clinical Modification (ICD-9-CM); Healthcare Common Procedure Coding System; and Current Procedural Terminology codes. Institutional review board approval was not required due to the de-identified nature of this existing data source and methods used by this database to protect both patients and health care sites.

\section{Patient Selection}

Adult patients with migraine $\geq 18$ years of age initiating their first PMM class (antidepressants, antiepileptics, beta blockers, or neurotoxins) from 2011 through 2013 (index date=first PMM class claim during the index period) were identified, with a 1-year pre-index clean period established for all PMMs (i.e., patients could not have received a PMM during the 12-month pre-index period). Patients in this parent cohort were required to have $\geq 2$ outpatient (or 1 inpatient) migraine diagnosis codes (ICD-9-CM 346.xx) from 1 year pre-index to 1 year post-index with at least 1 code occurring pre-index. The inclusion criteria also required 12 months of pre- and post-index continuous medical and prescription enrollment. Consistent with other research and to increase specificity, ${ }_{1}^{14}$ patients were excluded if, during the 12 months before the first claim for any PMM class (index or switched class), they had an ICD-9-CM code for a disease unrelated to migraine treated by that PMM class (e.g., epilepsy [345.xx] and antiepileptics; hypertension/congestive heart failure [401.xx-405.xx, 428.xx] and beta blockers; and depression [296.2x, 296.3x, 290.21, 292.84, 296.xx, 298.0, 300.4, 309.0, 309.1, 311.0, 311.x] and antidepressants). Other patient exclusion criteria were if they had a claim for $\geq 2$ classes of PMMs at index, or if they had a claim for a PMM within 14 days of initiation of another PMM class. In addition, patients with Medicaid coverage were excluded due to the heterogeneity of the patient population and a notably different health services and reimbursement structure compared with commercial providers. Patients with human immunodeficiency virus infection or cancer from 1 year pre-index to 1 year post-index were also excluded to reduce heterogeneity.

From the parent cohort of patients with migraine initiating their first PMM class, 4 mutually exclusive subgroups were compiled for subsequent analysis:

- Persistent (PMM-persistent): From index to +365 days postindex, patients had no gap 60 days or greater in their index class therapy, and from index to +365 days post-index, patients had no claim for a non-index class preventive migraine drug.

- Discontinued initial class or switched to a second class (PMM1): During the time period +14 days to +365 days post-index, patients had claims for exactly 1 non-index class PMM (this new medication class was subsequently continued without a 60 -day gap in treatment to +365 post-index), or in the time period from index to +365 days post-index, patients had a 60-day gap in their index-class therapy.

- Discontinued second class or switched to a third class (PMM2): During the time period +14 days to +365 days post-index, patients had claims for exactly 2 different non-index class PMMs (the final medication class was subsequently continued without the 60 -day gap in treatment to +365 days post-index), or in the time period from index to +365 days post-index, patients had a 60-day gap in their third-class therapy.

- Discontinued a third class or switched to a fourth class (PMM3): During the time period +14 days to +365 days post-index, patients had claims for 3 non-index class PMMs, or in the time period from index to +365 days post-index, patients had a 60-day gap in their second-class therapy. 
These switches were all class-level switches (classes: antiepileptic, beta blocker, antidepressant, or neurotoxin) and were assessed from 14 days post-drug initiation to 365 days postindex to reduce sample contamination by combination drug users or patients receiving drugs for another condition (clinical advice was such that true switches would not happen in under 14 days). Changes in PMMs within the same drug class were not identified as defining criteria for switching or discontinuation. A total of 264 patients were excluded because they could not be assigned to 1 of the 4 subgroups (e.g., quick switchers and combination drug users).

\section{Main Outcome Measures}

All-cause total direct costs (fully adjudicated claims) and HCRU were estimated for the 3 PMM subgroups versus the persistent subgroup in the 12 -month post-index period. Total all-cause direct health care costs included outpatient, inpatient, ED, and prescription costs. HCRU included number of outpatient visits, inpatient visits, and ED visits. Migraine-specific direct costs and HCRU were also determined using the subset of outpatient/inpatient/ED claims with an associated migraine diagnosis code (ICD-9-CM 346.xx in any position on the claim) or prescriptions determined to be migraine-specific (per treatment guidelines noted in the next paragraph).

PMM subgroup eligibility was approached as follows: (a) at the drug class-level, (b) specific to oral preventive treatments or neurotoxins, (c) included medications within the American Headache Society/American Academy of Neurology preventive treatment guidelines for episodic migraine, (d) specific to treatments with evidence of being established or probably effective per the guidelines (evidence levels A and B, respectively), and (e) only included treatments captured in a prescription claims database. ${ }^{15}$ Similarly, abortive migraine medications for managing headache (hereafter referred to as "acute medications") were analyzed at the drug class level based on guidelines for the pharmacologic treatment of acute attacks, levels A through C. ${ }^{16}$

\section{Statistical Analysis}

Bias and covariate imbalance were accounted for via the use of propensity scores (PS), which were calculated separately for each pairwise comparison between each different PMM subgroup versus the persistent subgroup using logistic regression. PS bin bootstrapping, ${ }^{17}$ controlling for patient baseline characteristic covariates at index (i.e., age, gender, insurance type/plan, geographical region, prescribing physician type, and index drug) and pre-index (total cost, comorbidities, and Charlson Comorbidity Index [CCI] $)^{18}$ was used to compare costs between each PMM and the persistent subgroup. Bootstrap simulations were performed ( $n=1,000$ to 5,000, depending on the comparison), resulting in PS-adjusted $P$ values for determining statistical difference between each PMM subgroup and the persistent subgroup. Patients missing more than 2 covariates were not assigned a PS and were excluded from that particular pairwise comparison.
Unadjusted costs and HCRU statistics were presented along with PS-adjusted $P$ values for comparisons. The overall significance level for cost and HCRU was $\alpha=0.05$, with any $P$ value less than that value being considered "statistically significant." HCRU percentages were calculated by dividing the number of patients with a visit by the total number of patients in the cohort. Summary descriptive analyses for baseline characteristics and acute medication use post-index were presented.

\section{Results}

\section{Patient Disposition, Demographics, and Pre-Index Comorbidities}

From the database, 6,238,123 patients were identified to have at least 1 claim for PMM from January 1, 2011, through December 31, 2013, with 12 months of continuous enrollment pre- and post-index (Figure 1). The final sample consisted of 55,402 patients, including 12,688 persistent, 37,665 PMM1, 4,687 PMM2, and 362 PMM3 patients (Table 1). Demographic and baseline medical characteristic data for each of the 4 subgroups were generally comparable, except that the persistent subgroup was slightly older (mean 41.1 years) and had a slightly higher proportion of females (86.8\%; Table 1). The PMM3 subgroup had a slightly lower mean CCI of 0.29 , a larger percentage of patients under the care of a neurologist (43.6\%), and lower rates of pre-index key comorbidities. The percentage of patients with at least 1 claim for chronic migraine during the pre-index period was highest in the PMM2 subgroup (6.3\%). Patterns of persistence versus $\geq 1$ switching or discontinuation by index preventive medication class included neurotoxins (35.6\% vs. $64.3 \%$ ); antiepileptics ( $23.8 \%$ vs. $76.2 \%$ ); beta blockers $(21.4 \%$ vs. $78.5 \%$ ); and antidepressants (18.3\% vs. $81.6 \%)$. Mean preindex total costs were similar across the persistent and PMM subgroups, ranging from $\$ 10,652$ to $\$ 11,075$.

\section{Post-Index Patterns of Acute Medication Use}

During the post-index period, $23.6 \%-31.8 \%$ of patients used at least 1 of the 6 acute medication classes (Figure 2). The most commonly filled prescription claims (referred to as "use") for acute medications were opioids (10.9\%-18.2\%) followed by triptans (5.8\%-8.0\%) and NSAIDs/acetaminophen (3.9\% to $5.1 \%$ ). Patients discontinuing their previous drug class or requiring more drug class switches appeared to have increased use of any acute medication, especially opioids. Use of any acute medication ranged from a mean of 10.7-15.9 days per month (Appendix A, available in online article). The PMM3 subgroup had the highest mean days per month (15.9) of any acute medications, followed by the persistent subgroup (13.4).

\section{Post-Index All-Cause HCRU and Costs}

Most patients ( $>99 \%$ ), regardless of subgroup, had at least 1 outpatient visit during the 1-year post-index period; however, the mean number of total outpatient visits in the post-index 


\section{FIGURE 1 Patient Selection and Flow}

Patients with at least 1 claim for PMM (NDC/HCPCS) $\mathrm{N}=6,238,123$

Patients with migraine claims (346.xx), 1 inpatient visit within 12 months pre-index, or 2 outpatient visits with first visit within 12 months pre-index and second visit within 12 months pre-index to 12 months post-index $\mathrm{n}=363,268$

Aged $\geq 18$ years $n=323,696$

Patients with at least 12 months pre- and post-index continuous enrollment $n=156,271$

Excluded patients with PMM claim 12 months pre-index $n=90,604$

Excluded patients with at least 2 PMM drug classes on index date $\mathrm{n}=89,648$

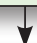

Excluded patients with HIV or cancer diagnosis 12 months pre-index to 12 months post-index

$$
\mathrm{n}=77,956
$$

Excluded patients with antiepilepsy index drug or claim 12 months postindex and epilepsy diagnosis 12 months before antiepilepsy drug claim $n=76,305$

Excluded patients with beta-blocker index drug or claim 12 months postindex and hypertension or congestive heart failure diagnosis 12 months before beta-blocker drug claim $\mathrm{n}=67,886$

Excluded patients with antidepressant index drug or claim 12 months postindex and depression diagnosis 12 months before antidepressant claim $\mathrm{n}=61,502$

Excluded patients with missing cost data $\mathrm{n}=61,496$

Excluded patients not assigned to one of the PMM-persistent subgroups $n=61,232$

\section{Excluded all Medicaid patients} $n=55,402$

\section{Subgroups}

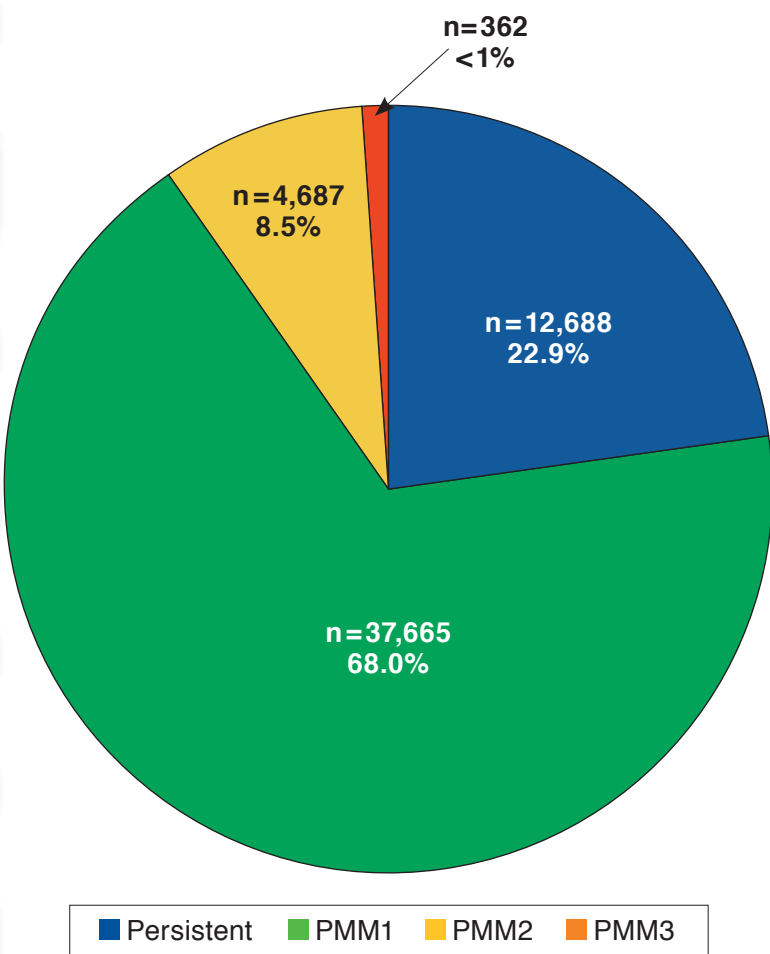

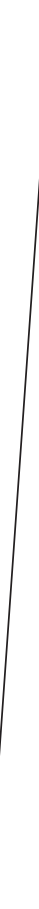

HCPCS = Healthcare Common Procedure Coding System; HIV = human immunodeficiency virus; NDC= National Drug Code; PMM = preventive migraine medication. 
TABLE 1 Demographic and Baseline Medical Characteristics at Index Date and Selected Pre-Index Comorbidities

\begin{tabular}{|c|c|c|c|c|c|}
\hline & $\begin{array}{l}\text { Total Sample } \\
(\mathrm{N}=55,402)\end{array}$ & $\begin{array}{c}\text { Persistent } \\
(\mathrm{n}=12,688)\end{array}$ & $\begin{array}{c}\text { PMM1 } \\
(\mathrm{n}=37,665)\end{array}$ & $\begin{array}{c}\text { PMM2 } \\
(n=4,687)\end{array}$ & $\begin{array}{c}\text { PMM3 } \\
(\mathrm{n}=362)\end{array}$ \\
\hline Percentage of total & 100 & 22.9 & 68.0 & 8.5 & $<1$ \\
\hline Mean (SD) age, years & $39.2(12.1)$ & $41.1 \quad(11.9)$ & $38.7 \quad(12.1)$ & $37.6 \quad(11.6)$ & $36.9 \quad(11.4)$ \\
\hline \multicolumn{6}{|l|}{ Age distribution, years, n (\%) } \\
\hline $18-24$ & $7,533 \quad(13.6)$ & $1,265 \quad(10.0)$ & $5,453 \quad(14.5)$ & $750 \quad(16.0)$ & $65 \quad(18.0)$ \\
\hline $25-34$ & $12,925 \quad(23.3)$ & $2,540 \quad(20.0)$ & $9,069 \quad(24.1)$ & $1,222 \quad(26.1)$ & $94 \quad(26.0)$ \\
\hline $35-44$ & $16,444 \quad(29.7)$ & $3,894 \quad(30.7)$ & $11,035 \quad(29.3)$ & $1,405 \quad(30.0)$ & $110 \quad(30.4)$ \\
\hline $45-54$ & $12,392 \quad(22.4)$ & $3,252 \quad(25.6)$ & $8,157 \quad(21.7)$ & $918 \quad(19.6)$ & $65 \quad(18.0)$ \\
\hline $55-64$ & $5,219 \quad(9.4)$ & $1,492 \quad(11.8)$ & $3,350 \quad(8.9)$ & $349 \quad(7.4)$ & $28 \quad(7.7)$ \\
\hline $65+$ & $889 \quad(1.6)$ & $245 \quad(1.9)$ & $601 \quad(1.6)$ & $43 \quad(0.9)$ & $0 \quad(0)$ \\
\hline Female gender, $\mathrm{n}(\%)$ & $46,792 \quad(84.5)$ & $11,014 \quad(86.8)$ & $31,550 \quad(83.8)$ & $3,929 \quad(83.8)$ & $299 \quad(82.6)$ \\
\hline \multicolumn{6}{|l|}{ Geographic region, n (\%) } \\
\hline Northeast & $6,612 \quad(11.9)$ & $1,578 \quad(12.4)$ & $4,458 \quad(11.8)$ & $547 \quad(11.7)$ & $29 \quad(8.0)$ \\
\hline North Central & $12,967 \quad(23.4)$ & $3,144 \quad(24.8)$ & $8,633 \quad(22.9)$ & $1,106 \quad(23.6)$ & $84 \quad(23.2)$ \\
\hline South & $22,493 \quad(40.6)$ & $4,973 \quad(39.2)$ & $15,491 \quad(41.1)$ & $1,869 \quad(39.9)$ & $160 \quad(44.2)$ \\
\hline West & $11,272 \quad(20.3)$ & $2,326 \quad(18.3)$ & $7,860 \quad(20.9)$ & $1,009 \quad(21.5)$ & $77 \quad(21.3)$ \\
\hline Unknown & $2,058 \quad(3.7)$ & $667 \quad(5.3)$ & $1,223 \quad(3.2)$ & $156 \quad(3.3)$ & $12 \quad(3.3)$ \\
\hline \multicolumn{6}{|l|}{ Insurance plan type, $\mathrm{n}(\%)$} \\
\hline Commercial & $54,090 \quad(97.6)$ & $12,317 \quad(97.1)$ & $36,789 \quad(97.7)$ & $4,622 \quad(98.6)$ & $362(100.0)$ \\
\hline \multicolumn{6}{|l|}{ Physician type, $\mathrm{n}(\%)^{\mathrm{a}}$} \\
\hline Neurologist & $12,261 \quad(33.2)$ & $2,871 \quad(34.9)$ & $8,160 \quad(32.3)$ & $1,121 \quad(34.5)$ & $109 \quad(43.6)$ \\
\hline Primary care & $14,694 \quad(39.7)$ & $3,093 \quad(37.6)$ & $10,221 \quad(40.5)$ & $1,289 \quad(39.6)$ & $91 \quad(36.4)$ \\
\hline Other non-neurologist specialist & $10,018 \quad(27.1)$ & $2,265 \quad(27.5)$ & $6,861 \quad(27.2)$ & $842 \quad(25.9)$ & $50 \quad(20.0)$ \\
\hline Charlson Comorbidity Index, b mean (SD) & $0.31 \quad(0.68)$ & $0.31 \quad(0.68)$ & $0.31 \quad(0.68)$ & $0.30 \quad(0.63)$ & $0.29 \quad(0.68)$ \\
\hline Pre-index chronic migraine diagnosis, $\mathrm{n}(\%)$ & $2,527 \quad(4.6)$ & $739 \quad(5.8)$ & $1,472 \quad(3.9)$ & $294 \quad(6.3)$ & $22 \quad(6.1)$ \\
\hline \multicolumn{6}{|c|}{ Pre-index comorbidities and other billable medical claims, $\mathrm{n}(\%, \%$ changec $)$} \\
\hline Anxiety & $9,092(16.4,+2.5)$ & $2,111(16.6,+2.1)$ & $6,211(16.5,+2.1)$ & $713(15.2,+6.4)$ & $57(15.7,+8.3)$ \\
\hline Depression & $8,529(15.4,+3.5)$ & $2,203(17.4,+3.5)$ & $5,761(15.3,+3.0)$ & $544(11.6,+6.6)$ & $21 \quad(5.8,+12.2)$ \\
\hline Sleep disturbance & $7,273(13.1,+1.8)$ & $1,733(13.7,+1.5)$ & $4,949(13.1,+1.4)$ & $548(11.7,+5.1)$ & $43(11.9,+10.8)$ \\
\hline Long-term use of other medications ${ }^{\mathrm{d}}$ & $5,429 \quad(9.8,+2.1)$ & $1,371(10.8,+2.1)$ & $3,591 \quad(9.5,+1.8)$ & $440 \quad(9.4,+4.2)$ & $27 \quad(7.5,+11.0)$ \\
\hline Hypertension & $5,094 \quad(9.2,+2.4)$ & $1,299(10.2,+2.2)$ & $3,516 \quad(9.3,+2.2)$ & $266 \quad(5.7,+3.6)$ & $(3.6,+4.7)$ \\
\hline Diabetes mellitus & $2,738 \quad(4.9,+0.2)$ & $615 \quad(4.8,+0.3)$ & $1,948 \quad(5.2,+0.1)$ & $163(3.5,+0.7)$ & $(3.3,+2.2)$ \\
\hline Obstructive sleep apnea & $2,401 \quad(4.3,+1.4)$ & $619(4.9,+1.3)$ & $1,614 \quad(4.3,+1.2)$ & $152(3.2,+2.6)$ & $(4.4,+3.0)$ \\
\hline Other headache types & $1,822 \quad(3.3,-0.1)$ & $425 \quad(3.3,-0.6)$ & $1,186 \quad(3.1,-0.3)$ & $196 \quad(4.2,+2.2)$ & $(4.1,+6.1)$ \\
\hline Cardiovascular disease & $4,917 \quad(8.9,-0.9)$ & $1,176 \quad(9.3,-1.3)$ & $3,334 \quad(8.9,-1.0)$ & $384(8.2,+0.2)$ & $(6.4,+3.3)$ \\
\hline Allodynia & $4,286 \quad(7.7,-0.5)$ & $912(7.2,-0.6)$ & $3,003 \quad(8.0,-0.9)$ & $346 \quad(7.4,+1.9)$ & $(6.9,+3.9)$ \\
\hline \multicolumn{6}{|l|}{ Index drug class, $\mathrm{n}(\%)^{\mathrm{e}}[\%]^{\mathrm{f}}$} \\
\hline Antiepileptic & $30,927 \quad(55.8)$ & $7,348(57.9)[23.8]$ & $21,351(56.7)[69.0]$ & $2,077(44.3) \quad[6.7]$ & $151(41.7) \quad[0.5]$ \\
\hline Antidepressant & $10,524 \quad(19.0)$ & $1,930(15.2) \quad[18.3]$ & $7,273(19.3)[69.1]$ & $1,204(25.7) \quad[11.4]$ & $117(32.3) \quad[1.1]$ \\
\hline Beta blocker & $11,007 \quad(19.9)$ & $2,361(18.6) \quad[21.4]$ & $7,391(19.6) \quad[67.1]$ & $1,178(25.1) \quad[10.7]$ & $77(21.3) \quad[0.7]$ \\
\hline Neurotoxin & $2,944 \quad(5.3)$ & 1,049 (8.3) $[35.6]$ & $1,650 \quad(4.4)[56.0]$ & $228 \quad(4.9) \quad[7.7]$ & $17(4.7)$ \\
\hline \multicolumn{6}{|l|}{ Disposition within each subgroup, $\mathrm{n}(\%)$} \\
\hline Switched & 4,805 & $(0.0)$ & $(11.0)$ & $(12.9)$ & $(14.9)$ \\
\hline Discontinued & $37,909 \quad(88.8)$ & $\begin{array}{ll}0 & (0.0) \\
\end{array}$ & $33,520 \quad(89.0)$ & $4,081 \quad(87.1)$ & $308 \quad(85.1)$ \\
\hline Pre-index total cost, USD & $10,773(19,198)$ & $11,020,(19,911)$ & $10,652(18,968)$ & $11,075(19,424)$ & $10,773(13,756)$ \\
\hline
\end{tabular}

a Identified by the provider claim at the time of or proximal to preventive initiation; ranked analysis (neurologist > primary care $>$ other specialist) conducted such that groups were mutually exclusive.

${ }^{b}$ Patients with missing scores were imputed as 0.

cPercentage point change in proportion of patients with at least 1 claim during the 12-month pre-index period to the 12-month post-index period for comorbidities known to be associated with migraine; those with $>2$ percentage point change in any subgroup were reported.

${ }^{d}$ Long-term (current) use of other medications for pain control that are considered high-risk, including methadone, opiate analgesic, and other high-risk medications.

${ }^{e}$ Number in parentheses indicates percentage within subgroup (column percent).

fNumber in brackets indicates percentage of specific medication group across subgroups (row percent).

$P M M=$ preventive migraine medication; $S D=$ standard deviation; $U S D=U . S$. dollars. 


\section{FIGURE 2 Post-Index Acute Medication Use}

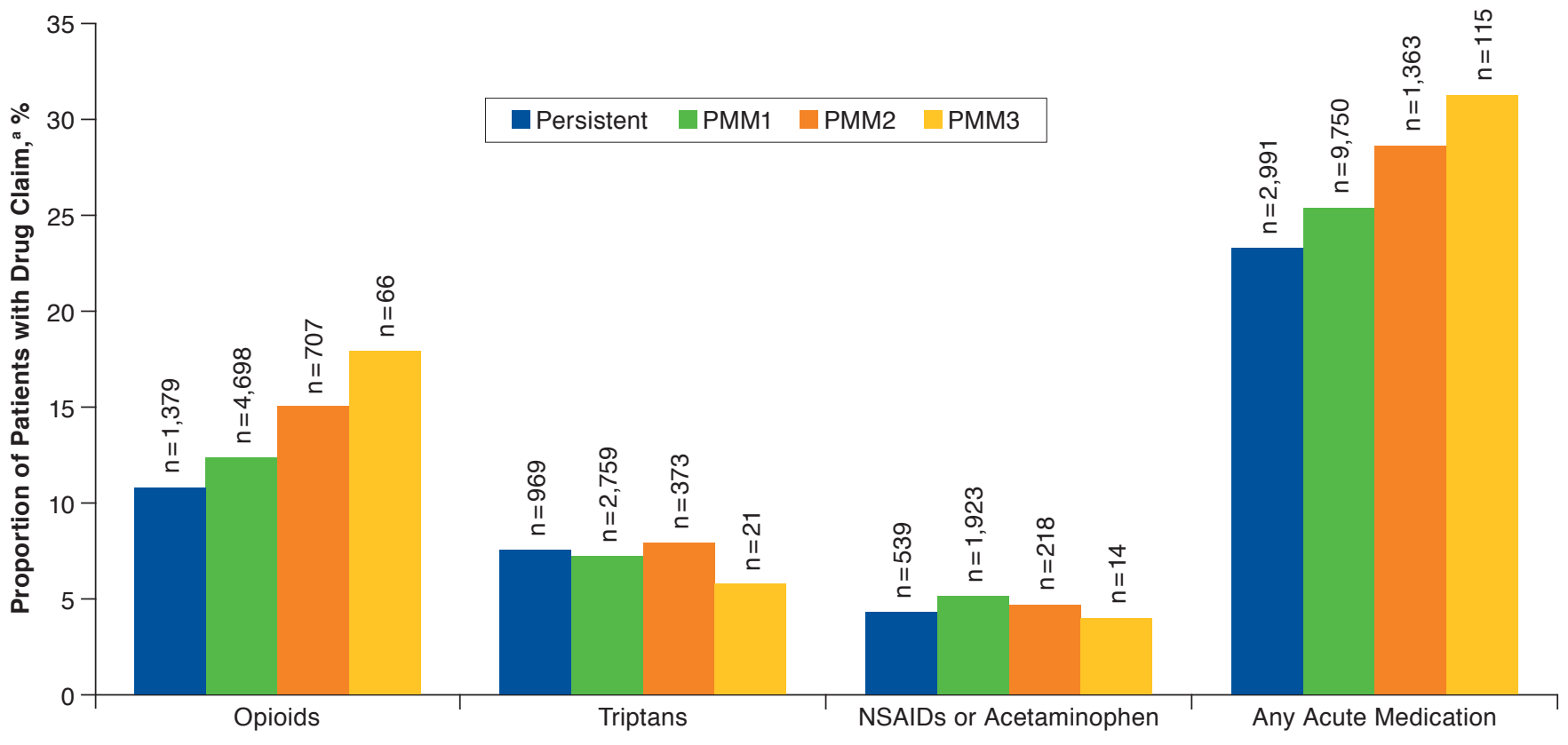

a Barbiturates, ergot alkaloids, and isometheptene and isometheptene combinations were all $\leq 3.6 \%$.

NSAIDs = nonsteroidal anti-inflammatory drugs; PMM= preventive migraine medication

period was higher for the PMM2 (2,570 visits per 100 patients; adjusted $P<0.001)$ and PMM3 subgroups $(3,132$ visits per 100 patients; adjusted $P<0.001)$ when compared with the persistent (2,237 visits per 100 patients) and PMMl subgroups (2,158 visits per 100 patients; Table 2). The persistent subgroup had a significantly lower percentage of patients with inpatient admissions (5.8\%) and ED visits (25.0\%) versus all PMM subgroups (7.8\%-13.5\% and 32.6\%-45.3\%, respectively; all adjusted pairwise comparisons, $P<0.001)$. Furthermore, the percentage of patients with inpatient admissions and ED visits was higher with each subsequent discontinuation or drug class switch. The persistent subgroup had, on average, significantly fewer annual mean inpatient admissions (8 visits per 100 patients) and ED visits (52 visits per 100 patients) than the PMM1, PMM2, and PMM3 subgroups (all adjusted pairwise comparisons, $P<0.001$; Table 2).

Mean all-cause unadjusted total direct costs derived from the post-index analysis showed significantly lower costs for the persistent $(\$ 11,941)$ subgroup versus the PMM2 $(\$ 13,429)$ and PMM3 $(\$ 18,394)$ subgroups (both adjusted pairwise comparisons, $P<0.001$; Figure 3 and Appendix B, available in online article). Mean all-cause total direct costs were lower for the PMMl $(\$ 11,239)$ versus the persistent subgroup (adjusted pairwise comparison, $P=0.548$ ). However, pharmacy costs were the second-largest component of total costs in most subgroups.
When pharmacy costs were excluded from total direct cost calculations, total costs were significantly lower in the persistent subgroup compared with all PMM subgroups, with the highest costs observed in the PMM3 subgroup $(\$ 15,230 ; P<0.001)$. A similar pattern of higher costs across the PMM subgroups was also observed for inpatient costs (mean range $\$ 1,798-\$ 3,444$ vs. $\$ 1,461$ for the persistent subgroup; all adjusted pairwise comparisons, $P \leq 0.014$ ) and ED costs (mean range $\$ 1,172$ $\$ 2,233$ vs. $\$ 815$ for the persistent subgroup; all adjusted pairwise comparisons, $P<0.001$ ).

\section{Migraine-Specific HCRU and Costs}

Most patients ( $\geq 89 \%$ ) had at least 1 migraine-specific outpatient visit during the 1-year post-index period. The PMM2 (97.0\%) and PMM3 (99.2\%) subgroups had significantly higher proportions of patients with migraine-specific outpatient visits versus the persistent subgroup (92.2\%; all adjusted pairwise comparisons, $P<0.001$ ). The mean number of annual migraine-specific outpatient visits was also significantly greater for the PMM2 (502 visits per 100 patients; adjusted $P<0.001$ ) and PMM3 (756 visits per 100 patients; adjusted $P<0.001$ ) subgroups compared with the persistent (357 visits per 100 patients) subgroup; however, the PMMl subgroup had significantly lower outpatient visits (305 per 100 patients; adjusted $P<0.001$ ) than the persistent subgroup (Table 2). The persistent subgroup 
Cycling Through Migraine Preventive Treatments: Implications for All-Cause Total Direct Costs and Disease-Specific Costs

TABLE 2 Post-Index Health Care Resource Utilization: All-Cause and Migraine-Specific

\begin{tabular}{|c|c|c|c|c|c|}
\hline & $\begin{array}{l}\text { Persistent } \\
(\mathrm{n}=12,688)\end{array}$ & $\begin{array}{c}\text { PMM1 } \\
(\mathbf{n}=37,665)\end{array}$ & $\begin{array}{c}\text { PMM2 } \\
(n=4,687)\end{array}$ & $\begin{array}{c}\text { PMM3 } \\
(\mathrm{n}=362)\end{array}$ & $\begin{array}{l}\text { Adjusted } \\
P \text { Value }^{\mathrm{a}}\end{array}$ \\
\hline \multicolumn{6}{|l|}{ All-cause HCRU (mean, SD) } \\
\hline Inpatient visits & $0.08 \quad(0.36)$ & $0.10 \quad(0.44)$ & $0.13 \quad(0.52)$ & $0.21 \quad(0.67)$ & all $<0.001$ \\
\hline Outpatient visits & $22.37 \quad(20.44)$ & $21.58 \quad(20.05)$ & $25.70 \quad(21.46)$ & $31.32(22.86)$ & $<0.001^{\mathrm{b}}$ \\
\hline Emergency department visits & $0.52 \quad(1.53)$ & $0.73 \quad(1.90)$ & $0.97 \quad(2.46)$ & $1.18 \quad(2.19)$ & all $<0.001$ \\
\hline \multicolumn{6}{|c|}{ Migraine-specific HCRU (mean, SD) } \\
\hline Inpatient visits & $(0.16)$ & $(0.19)$ & $(0.26)$ & $(0.46)$ & all $\leq 0.008$ \\
\hline Outpatient visits & $3.57 \quad(3.50)$ & $(3.67)$ & $(5.32)$ & $(5.97)$ & all $<0.001$ \\
\hline Emergency department visits & $0.16 \quad(0.83)$ & $(0.96)$ & $(1.21)$ & $(1.19)$ & all $<0.001$ \\
\hline
\end{tabular}

also had significantly lower rates of migraine-specific inpatient admissions (1.9\%) and migraine-specific ED visits (9.2\%) versus the PMM1, PMM2, and PMM3 subgroups (2.3\%-6.9\% and $12.1 \%-26.5 \%$, respectively; all adjusted pairwise comparisons, $P \leq 0.006$ ). In addition, the persistent subgroup had significantly fewer annual mean migraine-specific inpatient admissions (2.1 visits per 100 patients) and migraine-specific ED visits (16.1 visits per 100 patients) across the PMM subgroups (all adjusted pairwise comparisons, $P \leq 0.008$; Table 2).

Similar patterns were observed for disease-specific costs, with mean migraine-specific total direct costs being significantly lower for the persistent subgroup $(\$ 2,420)$ versus the PMM2 and PMM3 subgroups; higher costs were observed as the number of drug class switches or discontinuations increased (PMM1 \$1,894; PMM2 \$2,997; PMM3 \$5,004; all adjusted pairwise comparisons, $P<0.001$; Figure 3 and Appendix A). A similar trend in higher costs across all subgroups was also observed for inpatient costs (mean range \$198-\$690; all adjusted pairwise comparisons, $P \leq 0.03$ ) and ED costs (mean range \$151-\$659; all adjusted pairwise comparisons, $P<0.001$ ).

\section{Discussion}

Published data describing HCRU and cost burden are limited for patients with migraine who cycle through multiple preventive drug classes due to ineffectiveness or side effects. The purpose of this retrospective observational study was to describe demographic and medical characteristics, HCRU burden, direct costs, treatment patterns, and acute medication use over a 12 -month period in patients with migraine who discontinued their preventive medication or required 1,2 , or $\geq 3$ preventive drug class switches versus those who adhered persistently to their original medication class. Information gathered from the Truven Health Analytics MarketScan databases represents HCRU and direct costs among 55,402 adult patients with $\geq 2$ outpatient (or 1 inpatient) migraine diagnoses. Furthermore, this comprehensive annual HCRU and cost burden analysis used PS bin bootstrapping that controlled for patient baseline characteristic covariates. The subgroups were generally comparable in terms of demographic and clinical characteristics and are representative of a commercially insured population that was diagnosed with migraine and treated with a preventive medication. ${ }^{19}$

The distribution of antiepileptics, antidepressants, and beta blockers as first-line migraine preventive treatments was similar for the persistent and PMM1 subgroups. Within the PMM2 and PMM3 subgroups, the proportions initiating treatment with antiepileptics decreased, while the proportions initiating treatment with antidepressants and beta blockers increased relative to the other 2 subgroups. More research is needed to understand what drives the selection of the first and second PMM classes in patients with migraine, how that influences subsequent HCRU and cost burden, and whether or not treating both migraine and a concomitant condition with one drug intervention is in the best interest of patients, clinicians, and the health care system. ${ }^{3,20}$

Despite the use of PMMs, breakthrough migraine attacks often require acute treatment. ${ }^{21}$ Not surprisingly, use of acute medications during the 12-month post-index period was common (e.g., more than one fourth of our patients received an acute medication). Opioids (10.9\%-18.2\%), followed by triptans (5.8\%-8.0\%) and NSAIDs or acetaminophen (3.9\%-5.1\%), were the most frequently prescribed acute medications. Notably, a greater proportion of PMM3 patients had claims for opioids with more days of therapy versus subgroups of patients with fewer discontinuations or medication class switches. In contrast, triptan and NSAID or acetaminophen use appeared to slightly decrease among patients in the PMM3 subgroup. The reasons behind these changes in treatment patterns are unknown; however, these findings suggest a greater reliance on opioids as the patient progresses through multiple lines of preventive treatment. The slight decline in NSAID or triptan use may be indicative of a lack of therapeutic effect and possibly contraindications with concomitant medications or comorbid conditions. 


\section{FIGURE 3 Post-Index Direct Costs}

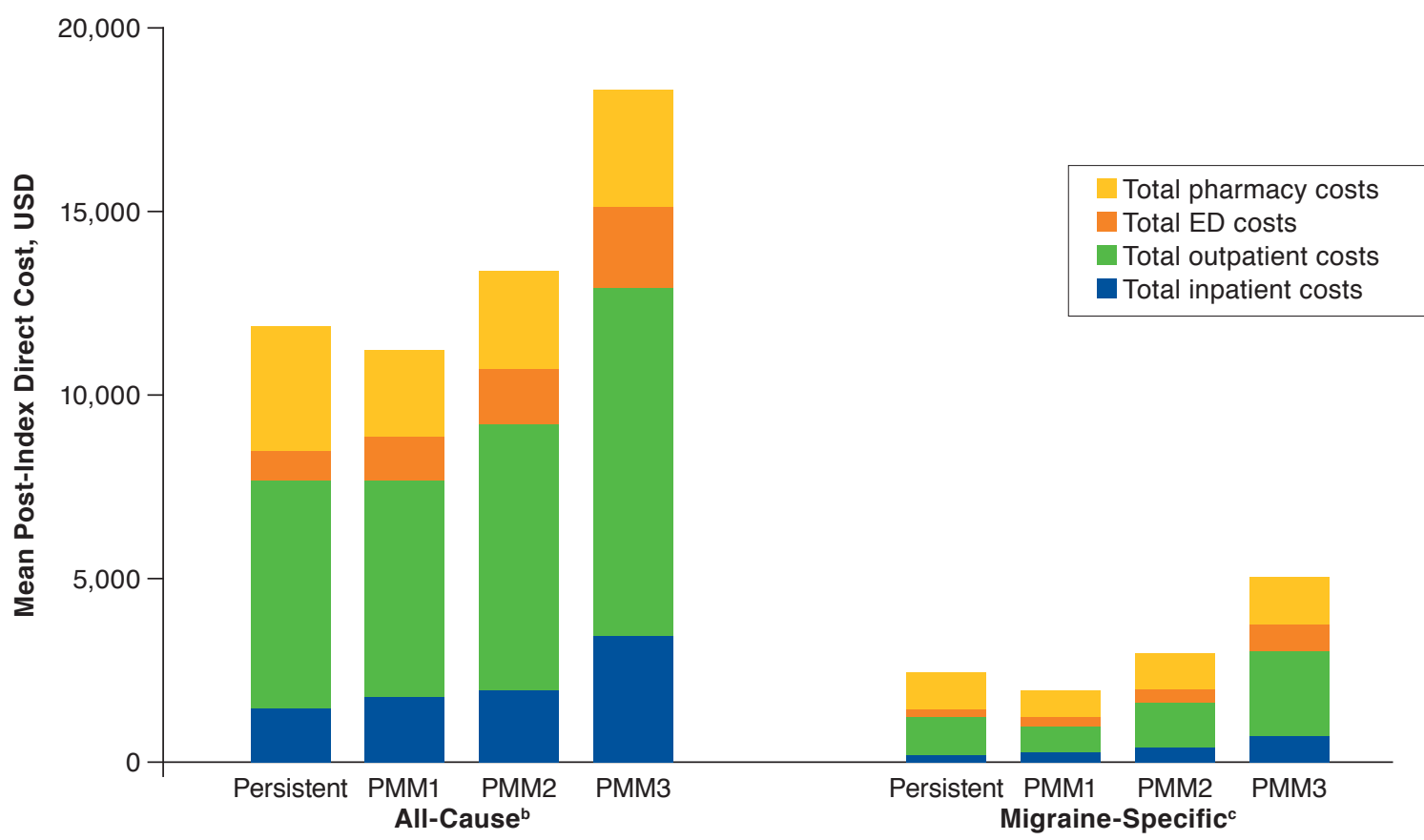

ap values based on adjusted data.

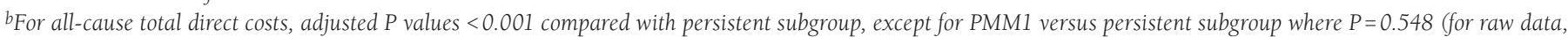
including SD, refer to Appendix A in online article).

${ }^{c}$ For migraine-specific total direct costs, adjusted P values are all <0.001 compared with the persistent subgroup (for raw data, including SD, refer to Appendix A). ED = emergency department; PMM= preventive migraine medication; USD =U.S. dollars.

Mean all-cause total direct costs in the PMM2 and PMM3 subgroups differed significantly from the persistent subgroup, with the former groups incurring $\$ 1,500$ to $\$ 6,500$ more costs per patient during the 12-month post-index period than the persistent group. Accordingly, this may translate to half a million dollars annually per 100 patients with $>1$ switch or discontinuation. Direct costs across the subgroups were largely attributed to outpatient visits ( $>50 \%$ ), followed by pharmacy costs (17\%-29\%) and inpatient costs (12\%-19\%). Although rates of inpatient admissions and ED visits comprised a lower proportion of HCRU than outpatient visits, the persistent subgroup had significantly lower rates of inpatient admissions (5.8\%) and ED visits (25.0\%) versus each PMM subgroup (7.8\%-13.5\% and $32.6 \%-45.3 \%$, respectively). This equates to 3 to 14 more inpatient visits and 21 to 66 more ED visits annually per 100 patients with $>1$ switch or discontinuation. Furthermore, the percentage of patients having inpatient admissions and ED visits increased notably with each subsequent discontinuation or medication class switch. The high rate of HCRU among switchers and discontinuers supports the belief that there is a remaining unmet treatment need among patients with migraine. It is unclear if reductions could be realized with the persistent use of an effective PMM in this population; it is worth noting that research suggests that the suboptimal use of available PMM is a risk factor for chronic migraine, ${ }^{22}$ for which there is evidence of a greater cost burden. ${ }^{23}$

We also observed that patients who remained persistent on their initial preventive medication class generally had higher pharmacy costs versus the subgroups who required medication switches. The explanation for the higher pharmacy costs is likely due to the nature of their being persistent on their preventive medication and the higher mean days of any acute medication per month. Because pharmacy costs may have confounded all-cause total costs, a second analysis was performed wherein prescription costs were removed. This analysis confirmed that there were significant and incrementally higher nonpharmacy total costs between the persistent subgroup and each PMM subgroup, with costs in the PMM3 subgroup being nearly double the amount of the persistent group. However, the continued heavy reliance on prescribed acute medications may be indicating sub-par efficacy for those with persistent use and possibly a reluctance or inability to switch to available alternative preventive treatments. 
A high cost burden was also observed in a separate analysis of migraine-specific direct costs among subgroups who cycle through multiple medication classes. Mean total migraine-specific direct costs were significantly less for the persistent subgroup versus the PMM2 and PMM3 subgroups, which incurred higher costs of $\$ 577-\$ 2,583$ per patient in the 12 -month post-index period as compared to persistent patients. Thus, escalating costs among the subgroup with medication class discontinuations or switches would account for an additional $\$ 57,700$-\$258,300 annually per 100 patients with $>1$ switch or discontinuation. Outpatient visits were the highest cost item across the subgroups. The method of disease-specific cost assessment used in this study has the potential to underrepresent migraine-specific costs and HCRU relative to all-cause estimates as our method relies on the presence of a migraine diagnosis code. Therefore, our method would not capture (a) costs attributed to comorbid conditions that are common in patients with migraine and (b) underuse of ICD-9-CM codes for migraine.

Few studies have investigated HCRU and cost burden for patients with migraine who cycle through multiple prevention medication classes. Hepp et al. (2016) used the same U.S.-based, health care claims database to investigate HCRU in patients with chronic migraine who were treated with onabotulinumtoxinA or oral migraine preventive medications. ${ }^{24}$ Similar to our study, they noted that patients receiving oral preventive treatments had higher headache-specific ED and inpatient visits during the 6- to 12 -month post-index periods. In contrast, treatment with onabotulinumtoxinA resulted in a significantly reduced number of ED and inpatient visits. Another study by Hepp et al. (2017), which also used the same U.S.-based database, reported that switching of oral preventive medications is very common among patients with chronic migraine. ${ }^{25}$ Furthermore, persistence with the initial medication was extremely low (i.e., $25 \%$ at 6 months and $14 \%$ at 12 months) and worsened as patients cycled through additional oral preventive medications. ${ }^{25}$ Our study of both patients with episodic and chronic migraine found that $>75 \%$ of patients switched or discontinued their index preventive treatment within 12 months.

\section{Limitations}

This study has some limitations to consider. The methods used to identify the study population have been shown to provide high specificity and sensitivity by using both a medical code for migraine and prescription claims for migraine treatment. ${ }^{26}$ However, due to the underuse of ICD-9-CM codes with greater specificity (i.e., 346.7x for chronic migraine) and the overuse of unspecified ICD-9-CM codes (i.e., 346.9x for migraine, unspecified), this study did not separate episodic and chronic migraine. ${ }^{27}$
Disease-specific costs may be underestimated due to requiring migraine-specific claims, even though other headacherelated claims may have been used to account for visits related to migraine. Importantly, the database provides no information on the following: disease severity, reasons for selecting the initial PMM class, rationale for changes in PMM treatment or discontinuation, and motives for not seeking an alternative preventive or if there was a lack of continued need when PMM was discontinued. It is possible that prescriber PMM class decisions were based on cost, or that switches or discontinuations were for reasons unrelated to clinical efficacy, such as formulary changes or an inability to pay for medications.

This study focused on changes at the drug class level instead of the individual drug level, which could be a limitation; however, only medications with evidence of being established or likely effective per treatment guidelines were included in this study. Another limitation is that medication use was based on prescription claims; actual patient consumption and specific indication for use were unknown. Over-the-counter treatments and alternative treatments for headache disorders were not captured in this database. Treatment patterns associated with contraindications were unknown. When comparing the cost data of PMM1 with that of the persistent group, the contribution of pharmacy costs versus a lack of continued treatment need within the PMMl group is not clear.

Finally, our study population may not be generalizable to all patients with migraine because only those with employeebased health insurance or Medicare supplemental insurance were included. Characteristics of migraine and its HCRU and cost patterns in the general population may be different from the population studied in claims data. This study was conducted in a fully adjudicated insurance claims data set; therefore, there are delays in availability of an analyzable data set when accounting for the duration of protocol design and conduct of the analyses. Additionally, the study required 1-year follow-up time for treatment analysis. Accordingly, changes in the health care system may have occurred by the time the study's results were reported.

\section{Conclusions}

This study demonstrates that patients with episodic or chronic migraine who cycle through multiple medication classes accrue more health care visits, ED and inpatient admissions, and acute medication use, a pattern notably higher among the subgroups with more than one switch or discontinuation annually. Antiepileptics were the most commonly prescribed migraine preventive treatment in this study; however, regardless of the first treatment choice, most patients had $\geq 1$ switch or discontinuation of treatment within a year. Further research is needed to understand if there are opportunities to decrease HCRU and direct costs in patients with migraine who switch or discontinue a migraine preventive treatment multiple times. 


\section{Authors}

JANET H. FORD, MPH, PhD; KRISTA SCHROEDER, PhD; ALLEN W. NYHUIS, MS; SHONDA A. FOSTER, PharmD; and SHEENA K. AURORA, MD, Eli Lilly and Company, Indianapolis, Indiana.

AUTHOR CORRESPONDENCE: Janet H. Ford, MPH, PhD, Eli Lilly and Company, Lilly Corporate Center, Indianapolis, IN 46285. Tel.: 317.903.8199; E-mail:ford_janet@lilly.com.

\section{DISCLOSURES}

Eli Lilly and Company was the sole sponsor and funder for this study and was responsible for the study design, data collection, data analysis, interpretation of data, and decision to publish the findings. All authors are employees and minor stockholders of Eli Lilly and Company. Nyhuis was employed by Eli Lilly and Company at the time of this study.

The findings of this study were presented in part at the 18th Congress of the International Headache Society; September 7-10, 2017; Vancouver, Canada.

\section{ACKNOWLEDGMENTS}

Writing support was provided by Teresa Tartaglione, PharmD (Synchrogenix, a Certara Company, Wilmington, DE).

\section{REFERENCES}

1. Raval AD, Shah A. National trends in direct health care expenditures among U.S. adults with migraine: 2004 to 2013. J Pain. 2017;18(1):96-107.

2. Buse DC, Manack AN, Fanning KM, et al. Chronic migraine prevalence, disability, and sociodemographic factors: results from the American Migraine Prevalence and Prevention Study. Headache. 2012;52(10):1456-70. 3. Silberstein SD, Holland S, Freitag F, Dodick DW, Argoff C, Ashman E. Evidence-based guideline update: pharmacologic treatment for episodic migraine prevention in adults: report of the Quality Standards Subcommittee of the American Academy of Neurology and the American Headache Society. Neurology. 2012;78(17):1337-45.

4. Holland S, Silberstein SD, Freitag F, Dodick DW, Argoff C, Ashman E. Evidence-based guideline update: NSAIDs and other complementary treatments for episodic migraine prevention in adults: report of the quality standards subcommittee of the American Academy of Neurology and the American Headache Society. Neurology. 2012;78(17):1346-53.

5. Lipton RB, Bigal ME, Diamond M, et al. Migraine prevalence, disease burden, and the need for preventive therapy. Neurology. 2007;68(5):343-49. 6. Peck KR, Johnson YL, Smitherman TA. Migraine. Handb Clin Neurol. 2016;138:283-93

7. Goldberg, LD. The cost of migraine and its treatment. Am J Manag Care. 2005;11(2 Suppl):S62-67.

8. Lipton RB, Stewart WF, Scher AI. Epidemiology and economic impact of migraine. Curr Med Res Opin. 2001;17(Suppl 1):S4-12.

9. $\mathrm{Wu}$ J, Hughes MD, Hudson MF, Wagner PJ. Antimigraine medication use and associated health care costs in employed patients. J Headache Pain. 2012;13(2):121-27.
10. Hepp Z, Bloudek LM, Varon SF. Systematic review of migraine prophylaxis adherence and persistence. J Manag Care Pharm. 2014;20(1):22-33. Available at: https://www.jmcp.org/doi/10.18553/jmcp.2014.20.1.22. 11. Schwedt TJ. Chronic migraine. BMJ. 2014;348:gl416.

12. Holland S, Fanning KM, Serrano D, Buse DC, Reed ML, Lipton RB. Rates and reasons for discontinuation of triptans and opioids in episodic migraine: results from the American Migraine Prevalence and Prevention (AMPP) study. J Neurol Sci. 2013;326(1-2):10-17.

13. Blumenfeld AM, Bloudek LM, Becker WJ, et al. Patterns of use and reasons for discontinuation of prophylactic medications for episodic migraine and chronic migraine: results from the second International Burden of Migraine Study (IBMS-II). Headache. 2013;53(4):644-45.

14. Shei A, Woolley JM, Desai PR, et al. Description of prophylactic drug utilization patterns in migraine patients. Value Health. 2015;18(3):A285.

15. Loder E, Burch R, and Rizzoli P. The 2012 AHS/AAN guidelines for prevention of episodic migraine: a summary and comparison with other recent clinical practice guidelines. Headache. 2012;52(6):930-45.

16. Matchar DB, Young WB, Rosenberg JH, et al. Evidence-based guidelines for migraine headache in the primary care setting: pharmacological management of acute attacks. U.S. Headache Consortium. 2000. Available at: http:// jasoncartermd.com/resources/pdf/Migraine\%20Guidelines.pdf. Accessed June 27, 2018.

17. Faries D, Peng X, Obenchain R. Costs and cost-effectiveness using propensity score bin bootstrapping. In: Faries DE, Leon AC, Haro JM, Obenchain RL, eds. Analysis of Observational Health Care Data Using SAS. Cary, NC: SAS Press; 2010.

18. Quan H, Sundararajan V, Halfon P, et al. Coding algorithms for defining comorbidities in ICD-9-CM and ICD-10 administrative data. Med Care. 2005;43(11):1130-39.

19. Smitherman TA, Burch R, Sheikh H, Loder E. The prevalence, impact, and treatment of migraine and severe headaches in the United States: a review of statistics from national surveillance studies. Headache. 2013;53(3):427-36.

20. Freitag FG, Shumate D. Current and investigational drugs for the prevention of migraine in adults and children. CNS Drugs. 2014;28(10):921-27. 21. Marmura MJ, Silberstein SD, Schwedt TJ. The acute treatment of migraine in adults: the American Headache Society evidence assessment of migraine pharmacotherapies. Headache. 2015;55(1):3-20.

22. Seng EK, Rains JA, Nicholson RA, Lipton RB. Improving medication adherence in migraine treatment. Curr Pain Headache Rep. 2015;19(6):24.

23. Lanteri-Minet M. Economic burden and costs of chronic migraine. Curr Pain Headache Rep. 2014;18(1):385.

24. Hepp Z, Rosen NL, Gillard PG, Varon SF, Mathew N, Dodick DW. Comparative effectiveness of onabotulinumtoxinA versus oral migraine prophylactic medications on headache-related resource utilization in the management of chronic migraine: retrospective analysis of a U.S.-based insurance claims database. Cephalalgia. 2016;36(9):862-74.

25. Hepp Z, Dodick DW, Varon SF, et al. Persistence and switching patterns of oral migraine prophylactic medications among patients with chronic migraine: a retrospective claims analysis. Cephalalgia. 2017;37(5):470-85. 26. Kolodner K, Lipton RB, Lafata JE, et al. Pharmacy and medical claims data identified migraine sufferers with high specificity but modest sensitivity. J Clin Epidemiol. 2004;57(9):962-72.

27. Hogan WR, Slee VN. Measuring the information gain of diagnosis vs. diagnosis category coding. AMIA Annu Symp Proc. 2010:306-10. Available at: https://www.ncbi.nlm.nih.gov/pmc/articles/PMC3041394/. Accessed June 27, 2018 
APPENDIX A Therapy per Month of Post-Index Acute Medication Use ${ }^{a}$

\begin{tabular}{|c|c|c|c|c|c|c|c|}
\hline & Opioids & Barbiturates & Triptans & $\begin{array}{l}\text { NSAIDs or } \\
\text { Acetaminophen }\end{array}$ & $\begin{array}{c}\text { Ergot Alkaloid } \\
\text { Derivative }\end{array}$ & $\begin{array}{l}\text { Isometheptene } \\
\text { and } \\
\text { Isometheptene } \\
\text { Combination } \\
\text { Agents }\end{array}$ & $\begin{array}{l}\text { Any Acute } \\
\text { Medication }\end{array}$ \\
\hline $\begin{array}{l}\text { Persistent } \\
(\mathrm{n}=12,688)\end{array}$ & $5.72 \quad(9.55)$ & $4.40 \quad(8.59)$ & $11.42 \quad(9.63)$ & $8.04 \quad(8.30)$ & $1.16 \quad(2.27)$ & $2.75 \quad(3.32)$ & $13.39(10.90)$ \\
\hline $\begin{array}{l}\text { PMMl } \\
(\mathrm{n}=37,665)\end{array}$ & $5.32 \quad(9.13)$ & $2.99 \quad(4.56)$ & $8.52 \quad(8.86)$ & $5.56 \quad(6.33)$ & $1.08 \quad(2.60)$ & $1.58 \quad(1.45)$ & $10.73(10.20)$ \\
\hline $\begin{array}{l}\text { PMM2 } \\
(n=4,687)\end{array}$ & $5.78 \quad(9.16)$ & $4.75 \quad(6.91)$ & $9.76 \quad(8.98)$ & $6.44 \quad(6.84)$ & $0.82 \quad(1.22)$ & $1.67 \quad(0.00)$ & $12.89(10.37)$ \\
\hline $\begin{array}{l}\text { PMM3 } \\
(n=362)\end{array}$ & $7.51 \quad(9.25)$ & $0.83 \quad(0.00)$ & $8.50(10.04)$ & $5.85 \quad(6.50)$ & $0.76 \quad(2.15)$ & - & $15.90(10.40)$ \\
\hline
\end{tabular}

aAll values are shown as mean $(S D)$.

NSAIDs = nonsteroidal anti-inflammatory drugs; $P M M=$ preventive migraine medication; $S D=$ standard deviation . 


\section{APPENDIX B Total Direct USD Costs: All-Cause and Migraine-Specific}

\begin{tabular}{|c|c|c|c|c|}
\hline & Persistent & PMM1 & PMM2 & PMM3 \\
\hline All-cause costs ${ }^{b}$ & $\mathrm{n}=12,688$ & $\mathrm{n}=37,665$ & $\mathrm{n}=4,687$ & $\mathrm{n}=362$ \\
\hline \multicolumn{5}{|l|}{ Total direct costs } \\
\hline Post-index unadjusted cost (USD) & $11,941(23,605)$ & $11,239(20,421)$ & $13,429(20,057)$ & $18,394(26,912)$ \\
\hline Post-index adjusted cost (USD) & $11,470 \quad(209)$ & $11,365 \quad(107)$ & $13,943 \quad(303)$ & $19,551 \quad(1,359)$ \\
\hline Adjusted $P$ value ${ }^{c}$ & - & 0.548 & $<0.0001$ & $<0.001$ \\
\hline \multicolumn{5}{|c|}{ Total direct costs minus prescription costs } \\
\hline Post-index unadjusted cost (USD) & $8,531(21,545)$ & $8,891(18,746)$ & $10,726(18,550)$ & $15,230(25,697)$ \\
\hline Post-index adjusted cost (USD) & $8,261 \quad(192)$ & $8,955 \quad(97)$ & $10,970 \quad(278)$ & $15,337 \quad(1,251)$ \\
\hline Adjusted $P$ value ${ }^{\mathrm{c}}$ & - & 0.002 & $<0.001$ & $<0.001$ \\
\hline \multicolumn{5}{|l|}{ Total inpatient direct costs } \\
\hline Post-index unadjusted cost (USD) & $1,461 \quad(10,312)$ & $1,798(11,643)$ & $1,985(11,436)$ & $3,444(15,974)$ \\
\hline Post-index adjusted cost (USD) & $1,497 \quad(96)$ & $1,800 \quad(60)$ & $2,066 \quad(173)$ & $3,143 \quad(691)$ \\
\hline Adjusted $P$ value ${ }^{c}$ & - & 0.003 & $<0.001$ & 0.014 \\
\hline \multicolumn{5}{|l|}{ Total outpatient direct costs } \\
\hline Post-index unadjusted cost (USD) & $6,255(15,005)$ & $5,921 \quad(10,133)$ & $7,272 \quad(9,782)$ & $9,553(10,847)$ \\
\hline Post-index adjusted cost (USD) & $5,918 \quad(130)$ & $5,999 \quad(51)$ & $7,464 \quad(135)$ & $9,993 \quad(587)$ \\
\hline Adjusted $P$ valuec & - & 0.600 & $<0.001$ & $<0.001$ \\
\hline \multicolumn{5}{|l|}{ Total emergency department costs } \\
\hline Post-index unadjusted cost (USD) & $815 \quad(3,216)$ & $1,172 \quad(4,332)$ & $1,473 \quad(3,868)$ & $2,233 \quad(4,998)$ \\
\hline Post-index adjusted cost (USD) & $846 \quad(28)$ & $1,156 \quad(21)$ & $1,438 \quad(58)$ & $2,181 \quad(259)$ \\
\hline Adjusted $P$ value ${ }^{c}$ & - & $<0.001$ & $<0.001$ & $<0.001$ \\
\hline \multicolumn{5}{|l|}{ Total pharmacy costs } \\
\hline Post-index unadjusted cost (USD) & $3,410 \quad(6,971)$ & $2,348 \quad(5,984)$ & $2,703 \quad(4,940)$ & $3,164 \quad(4,351)$ \\
\hline Post-index adjusted cost (USD) & $3,217 \quad(59)$ & $2,414 \quad(31)$ & $2,973 \quad(74)$ & $4,194 \quad(294)$ \\
\hline Adjusted $P$ value ${ }^{\mathrm{c}}$ & - & $<0.001$ & $<0.001$ & 0.012 \\
\hline Migraine-specific costs ${ }^{b}$ & $\mathrm{n}=12,688$ & $\mathbf{n}=37,665$ & $\mathbf{n}=4,687$ & $\mathrm{n}=362$ \\
\hline \multicolumn{5}{|l|}{ Total direct costs } \\
\hline Post-index unadjusted cost (USD) & $2,420 \quad(4,510)$ & $1,894 \quad(4,812)$ & $2,997 \quad(5,221)$ & $5,004 \quad(6,580)$ \\
\hline Post-index adjusted cost (USD) & $2,216 \quad(38)$ & $1,934 \quad(24)$ & $3,109 \quad(84)$ & $5,351 \quad(327)$ \\
\hline Adjusted $P$ value ${ }^{c}$ & - & $<0.001$ & $<0.001$ & $<0.001$ \\
\hline \multicolumn{5}{|c|}{ Total direct costs minus prescription costs } \\
\hline Post-index unadjusted cost (USD) & $1,390 \quad(3,854)$ & $1,219 \quad(3,854)$ & $2,045 \quad(4,754)$ & $3,716 \quad(5,946)$ \\
\hline Post-index adjusted cost (USD) & $1,254 \quad(33)$ & $1,237 \quad(19)$ & $2,074 \quad(77)$ & $3,842 \quad(314)$ \\
\hline Adjusted $P$ value & - & 0.626 & $<0.001$ & $<0.001$ \\
\hline \multicolumn{5}{|l|}{ Total inpatient direct costs } \\
\hline Post-index unadjusted cost (USD) & $198 \quad(2,586)$ & $253 \quad(2,794)$ & $354 \quad(3,286)$ & $690 \quad(3,393)$ \\
\hline Post-index adjusted cost (USD) & $195 \quad(22)$ & $252 \quad(14)$ & $332 \quad(49)$ & $680 \quad(166)$ \\
\hline Adjusted $P$ value ${ }^{\mathrm{c}}$ & - & 0.030 & 0.014 & 0.004 \\
\hline \multicolumn{5}{|l|}{ Total outpatient direct costs } \\
\hline Post-index unadjusted cost (USD) & $1,041 \quad(2,395)$ & $760 \quad(2,005)$ & $1,315 \quad(2,190)$ & $2,367 \quad(3,532)$ \\
\hline Post-index adjusted cost (USD) & $907 \quad(19)$ & $781 \quad(10)$ & $1,380 \quad(31)$ & $2,502 \quad(197)$ \\
\hline Adjusted $P$ valuec & - & $<0.001$ & $<0.001$ & $<0.001$ \\
\hline \multicolumn{5}{|l|}{ Total emergency department costs } \\
\hline Post-index unadjusted cost (USD) & $151 \quad(1,120)$ & $206 \quad(1,341)$ & $375 \quad(1,857)$ & $659 \quad(2,335)$ \\
\hline Post-index adjusted cost (USD) & $153 \quad(10)$ & $204 \quad(6)$ & $362 \quad(26)$ & $660 \quad(112)$ \\
\hline Adjusted $P$ value ${ }^{\mathrm{c}}$ & - & $<0.001$ & $<0.001$ & $<0.001$ \\
\hline \multicolumn{5}{|l|}{ Total pharmacy costs } \\
\hline Post-index unadjusted cost (USD) & $1,031 \quad(2,050)$ & $675 \quad(2,669)$ & $(1,724)$ & $1,288 \quad(1,684)$ \\
\hline Post-index adjusted cost (USD) & $962 \quad(16)$ & $697 \quad(14)$ & $1,035 \quad(27)$ & $1,509 \quad(99)$ \\
\hline Adjusted $P$ value ${ }^{c}$ & - & $<0.001$ & 0.320 & $<0.001$ \\
\hline \multicolumn{5}{|c|}{$\begin{array}{l}\text { a All values are shown as mean (SD). } \\
\text { bCosts were adjusted based on the } 2014 \text { Consumer Price Index. } \\
{ }^{c} \text { All pairwise comparisons were performed using propensity score-adjusted bin bootstrapping and compared with persistent subgroup. } \\
\text { PMM = preventive migraine medication; } S D=\text { standard deviation; USD =U.S. dollars. }\end{array}$} \\
\hline
\end{tabular}

